\title{
L'IMPLICATION PERSONNELLE: UN OUTIL PSYCHOSOCIAL POUR COMPRENDRE LE LIEN POPULATION-OBJET
}

\author{
Chloé Gurrieri \\ Rafael Pecly Wolter \\ Estelle Sorribas
}

\begin{abstract}
RESUMÉ. Le but de cet article est de présenter l'implication personnelle en tant qu'outil permettant une meilleure compréhension des relations entre un objet et une population. Dans cette étude, nous avons pris en compte deux variables indépendantes : la nationalité Brésilienne ou Française) et la religion (catholique ou sans religion). La mesure utilisée a été l'implication des participants par rapport à un objet fictif (une distribution de repas effectuée par l'Eglise). Les résultats indiquent que nos deux variables indépendantes (appartenance nationale et religieuse) n'ont pas le même effet sur chacune des dimensions de l'implication (la proximité, la valorisation de l'objet et la possibilité perçue d'action). Au vu de ces résultats, on a discuté l'intérêt de cette mesure pour une meilleure compréhension des phénomènes sociaux.
\end{abstract}

Mots-clef: implication, pensée sociale, approche structurale.

\section{A IMPLICAÇÃO PESSOAL: UM INSTRUMENTO PSICOSSOCIAL PARA COMPREENDER A RELAÇÃO POPULAÇÃO-OBJETO}

RESUMO. O objetivo deste artigo é apresentar a implicação pessoal como instrumento para uma melhor compreensão das relações entre um objeto e uma determinada população. Este estudo tem duas variáveis independentes, a nacionalidade (franceses e brasileiros) e a religião (católicos e pessoas sem religião). A medida usada neste estudo foi a implicação dos participantes relativa a um objeto fictício (uma distribuição de refeições feita pela Igreja). Os resultados mostram que as duas variáveis independentes (religião e nacionalidade) não influenciam da mesma maneira as três dimensões da implicação pessoal (a proximidade, a valorização do objeto, e a capacidade percebida de ação). A partir dos resultados discutimos o interesse desta medida para uma melhor compreensão dos fenômenos sociais.

Palavras-chave: implicação, pensamento social, abordagem estrutural.

\section{PERSONAL INVOLVEMENT: A PSYCHO-SOCIAL TOOL FOR UNDERSTANDING THE POPULATION-OBJECT RELATIONSHIP}

RESUMO. Personal involvement for a better understanding of the relationship between an object and a population is provided. Analysis implies in two independent variables, namely, nationality (French and Brazilian) and religion (Catholics and people without any religion). Parameter consisted of the participants' involvement with regard to a fictitious object (meals distributed by church people). Results show that independent variables (religion and nationality) failed to affect similarly the three dimensions of personal involvement (closeness, valorization of object and the act's perceived capacity). Interests on this issue were discussed for a better understanding of social phenomena.

Key words: Involvement, social thought, structural approach.

\section{LA IMPLICACIÓN PERSONAL: UN INSTRUMENTO PSICO-SOCIAL PARA COMPRENDER A RELACIÓN POBLACIÓN-OBJETO}

RESUMEN. El objetivo de este artículo es el de presentar la implicación personal como instrumento para una mejor comprensión de las relaciones entre un objeto y una determinada población. Este estudio tiene dos variables independientes:

* D.E.A. en Psychologie Sociale et Environnementale. Professor da Université Paris 5, Descartes. Institut de Psychologie, Centre Henri Piéron, Laboratoire de Psychologie Environnementale, Centre National de la Recherche Scientifique-(CNRS).

\# Mestre em Individu Social et Environnement. Professor da Université Paris 5, Descartes. Institut de Psychologie, Centre Henri Piéron, Laboratoire de Psychologie Environnementale, Centre National de la Recherche Scientifique-(CNRS). 
la nacionalidad (franceses y brasileños) y a religión (católicos y personas sin religión). La medida usada para el estudio fue la de la implicación de los participantes relativo a una situación ficticia (una distribución de comidas hecha por la Iglesia). Los resultados muestran que las dos variables independientes (religión y nacionalidad) no influencian de la misma manera las tres dimensiones de la implicación personal (la proximidad, a valorización del objeto, y la capacidad percibida de acción). A partir de los resultados, se discute el interés de esta medida para una mejor comprensión de los fenómenos sociales.

Palabras-clave: implicación personal, pensamiento social, abordaje estructural.

Souvent opposée à la pensée scientifique, la pensée sociale serait caractérisée par l'insuffisance et l'irrationalité, ainsi que par des erreurs et biais de tous genres. Par contre, la pensée scientifique serait performante et efficace. Cette antinomie semble aujourd'hui dépassée. Pour Rouquette (1998b), ces biais et ces erreurs font corps et sens. Etant donné que les processus cognitifs qui les sous-tendent ne se déroulent pas au hasard et sont liés les uns aux autres, on peut dire qu'ils font corps. En outre ils font également sens, car leur "occurrence, à propos d'un objet donné, est presque toujours la signature d'une position sociale particulière" (p. 37). La pensée sociale ne peut pas être réduite à une pensée biaisée : elle est plutôt une "pensée différente" (Guimelli, 1999, p. 18), ayant sa logique particulière.

La pensée sociale est définie par Guimelli (1999) comme une pensée autonome et spécifique, indépendante de la pensée rationnelle, mais cohabitant avec elle chez les mêmes individus; chacun de ces deux types de pensée interviendrait dans des contextes sociaux particuliers. Ces deux modes de pensée coexistent donc chez les individus et, selon le cas, l'un ou l'autre de ces modes de pensée sera activé. Rouquette (1998b) y distingue deux aspects complémentaires: la pensée, prenant pour objet "la réalité sociale" et celle qui se rapporte "à l'intervention de facteurs sociaux dans la réalisation commune de la pensée" (p. 33). D'après lui, la pensée sociale est élaborée en situation par les acteurs et les témoins de tous les aspects de la sociabilité.

Le but de cet article est de présenter l'implication comme un outil permettant d'étudier les relations entre un objet et un individu ou une population.

Nous présenterons cet outil en l'utilisant comme mesure permettant de quantifier les différences entre des groupes de participants par rapport à une problématique commune qui leur a été présentée dans la phase empirique. Cette mesure d'implication personnelle, qui intéresse différents publics en France, reste encore méconnue au Brésil. Cette mesure présente l'avantage de conjuguer trois différentes dimensions de l'implication, c'est-à-dire de prendre en considération les trois facteurs susceptibles d'expliquer l'implication des participants par rapport à un objet: la proximité perçue par rapport à cet objet, sa valorisation et la possibilité d'action perçue que le participant pense avoir par rapport à cet objet.

Dans cet article, nous souhaitons mettre en évidence les variations d'implication par rapport à une problématique identique pour tous les participants, des variations qui seraient dues à l'effet des prises de position sociales des participants, à savoir leur appartenance nationale et leur sentiment d'appartenance à une religion.

Ces deux appartenances peuvent notamment être ramenées, d'un point de vue psychosocial, à des appartenances idéologiques. Or, nous savons que l'idéologie doit jouer un rôle certain au niveau de la perception de la réalité.

\section{L'IDEOLOGIE}

L'idéologie permet de donner un sens à la réalité, c'est un ensemble plus ou moins organisé de "représentations et d'explications du monde et, notamment, du monde des interactions sociales, où le mobile premier n'est pas d'abord d'ordre vérificateur" (Deconchy, 1989, pp 237). "Les idéologies comportent un ensemble, faiblement systématisé, de principes organisateurs de notre "vision du monde". Ces principes sont de simples repères, plus ou moins flous et parfois contradictoires, à partir desquels le sujet doit construire activement le sens des réalités auxquelles il est confronté" (Ibanez, 1999, pp 333).

L'idéologie aurait essentiellement trois fonctions :

- Une fonction organisatrice des attitudes et donc, de la "vision du monde", propre aux individus.

- Une fonction de maintien d'une cohérence interne qui permet de ressentir un sentiment "d'unité".

- Une fonction de catégorisation de la réalité tenant compte de l'adéquation de cette dernière à la "vision du monde", propre aux individus.

Il convient, dans ce cadre, de rappeler que les représentations sociales, porteuses de déterminants et 
d'enjeux sociaux, propres aux différents groupes, ont justement leur place en aval de l'idéologie. Elles seraient, en particulier, l'expression d'une idéologie rattachée à un objet donné. En effet, l'idéologie structure les représentations sociales propres aux différents groupes.

Rouquette, dans Représentations et idéologie (1996), montre, entre autres, que les différentes transmissions et interprétations d'un message dans une chaîne d'individus sont bien dues au positionnement initial des participants par rapport au thème présenté dans le message. Ces résultats viennent étayer la démarche de comparaison entreprise dans cet article.

En effet, en partant d'une vision "sociale" du phénomène d'adhésion à des croyances religieuses, qui seraient avant tout le fait d'une société particulière (plutôt qu'une réalité intime et individuelle), nous pouvons parler d'une "appartenance idéologique religieuse".

En particulier, d'après Deconchy (1989), par "idéologie", il faudrait comprendre une forme de la pensée sociale qui se situerait au même niveau que les représentations (qui serait, en somme, plutôt propre à des groupes sociaux qu'à toute une nation), mais déclinée selon des modalités différentes (c'est-à-dire, liée à un ensemble de croyances d'un groupe). Nous pouvons citer comme exemples de cette forme d'idéologie ${ }^{1}$, notamment, l'idéologie politique ou religieuse qui oppose des groupes sociaux ayant des appartenances idéologiques différentes.

D'après Rouquette (1996), en accord avec Deconchy, l'idéologie n'a pas de véritable consistance théorique. Cette notion paraît, de fait, ambivalente : elle aurait une acception négative, en tant que savoir et manière de penser irrationnelle des masses, et une acception positive, en tant qu'élément générateur de cohésion et de capacité d'adaptation au sein des groupes (Rouquette, 1996). Les idéologies "vont donc regrouper aussi bien des croyances, des valeurs, des attitudes et des comportements que des façons de percevoir et de penser qui font l'objet d'un accord au point de constituer un ensemble de normes pour une société, lui dictant ce qui est souhaitable et ce qu'il faudrait faire" (Jowett \& O' Donnel, cités par Rouquette, 1996, p.165). Pour essayer de la définir : "l'idéologie est ce qui rend un ensemble de croyances, d'attitudes et de représentations, à la fois possibles et compatibles au sein d'une population"

1 Par opposition à des idéologies propres à une société qui seraient des «Représentations collectives ».
(Rouquette 1996, p.167). En ce sens, l'idéologie se situerait à un niveau supérieur par rapport aux représentations, mais elle ne serait pas univoque dans une société donnée, parce que considérée comme une forme de la pensée sociale, se déclinant de manière différente d'un groupe à l'autre (pour reprendre notre exemple précédent, il s'agit d'être politiquement plutôt à gauche ou plutôt à droite, d'être catholique ou athé). Les idéologies seraient, en particulier d'après Rouquette, des "machines à interpréter". C'est dans ce cadre de référence particulier que l'idéologie est abordée dans cette étude.

Compte tenu de ces considérations, il paraît pertinent de se questionner sur le rôle des différentes appartenances idéologiques (ici nationales et religieuses) [partie supprimée] sur le niveau d'implication engendré par un message identique chez les différents sous-groupes de participants, qui contrastent idéologiquement entre eux.

\section{L'IMPLICATION PERSONNELLE.}

L'implication personnelle (Rouquette, 1988 ; 1997; 1998a ; 1998b; 1998c; Guimelli, 2002 ; Flament \& Rouquette, 2003), introduite par Rouquette, pourrait être définie (Gruev-Vintila, 2005) comme l'expression individuelle et socialement déterminée du lien entre l'individu et l'objet sur lequel il porte son attention. Ce facteur d'implication peut donc être envisagé comme "une variable explicative majeure de la pensée sociale" (Guimelli, 1998, p. 84), et présente l'avantage de pouvoir également se définir de manière opérationnelle (Rouquette, 1997), ce qui élargit son applicabilité à de nombreux champs théoriques et recherches empiriques. L'implication personnelle jouerait, surtout, un "rôle fondamental dans l'expression et la dynamique des représentations sociales" (Baggio, 2006, p.16) et, c'est cela qui en fait un facteur dont la prise en compte apparaît désormais primordiale dans l'analyse des processus intervenant dans l'organisation des formes de la pensée sociale et, en particulier, des représentations sociales. En effet, l'implication interviendrait sur la formation et la structuration des représentations (Flament \& Rouquette, 2003) et pourrait aussi, à son tour, être affectée par la nature de ces dernières (Baggio, 2006 ; Gruev-Vintila, 2005).

Si nous reprenons la définition de la psychologie sociale, donnée par Moscovici (1984), où le moi (Ego), l'autre (Alter) et l'objet forment une triade, 
l'implication s'insèrerait dans cette triangulation en tant que médiateur de la relation Ego-Objet-Alter:

\section{Ego}

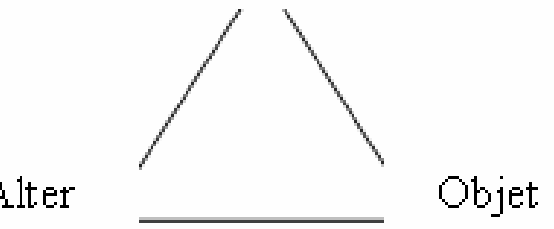

L'implication personnelle agirait essentiellement entre Ego et Objet ; toutefois, prise au sens large, cette variable médiatrice est également susceptible d'intervenir entre Ego et Alter et Alter et Objet; par exemple, le niveau d'implication des membres du groupe d'appartenance (Alter) d'un individu par rapport à un objet va certainement jouer sur la manière comment cet individu (Ego) se sentira impliqué par rapport à ce même objet.

L'implication est composée de trois dimensions indépendantes entre elles (cf., par ex., Flament \& Rouquette, 2003; Rouquette, 1997), ce qui signifie que le niveau d'implication par rapport à une des dimensions ne permet d'aucune manière de prévoir le niveau d'implication des mêmes personnes par rapport aux deux autres dimensions. La première dimension, la proximité $^{2}$, concerne la proximité perçue à l'objet, au niveau "d'adéquation personnelle estimée relativement à l'objet" (Flament \& Rouquette, 2003, p.127). Cette dimension peut être mesurée par l'échelle de Likert (dans cette recherche, il s'agissait d'une échelle en 6 points) allant de "cela me concerne à titre personnel" jusqu'à "cela ne me concerne pas plus que n'importe qui".

La deuxième dimension, la mise en valeur de l'objet, se rattache à l'importance de l'objet pour une personne ou un groupe; il s'agit, en quelque sorte, de l'estimation de l'enjeu attaché à l'objet. Cette dimension correspond à une échelle pouvant aller du pôle "c'est une question de vie ou de mort" à "c'est une question sans importance" (échelle de Likert, ici en 6 points).

La dernière dimension, la possibilité perçue d'action, traduit la position perçue du sujet par rapport à l'objet: soit l'individu se voit comme un agent et pense que son action va avoir un certain poids sur les

2 Cette dimension est souvent appelée, dans la littérature, "identification personnelle". Nous avons préféré dans ce cadre, pour plus de clarté, le terme "proximité". évènements soit, au contraire, il se pose comme patient et croit ne rien pouvoir changer. Cette dimension peut être mesurée par une échelle exprimant la graduation allant de l'impuissance au contrôle total perçu (échelle de Likert, ici, en 6 points).

Dans le cadre de cette étude, nous pensons que ces trois mesures de l'implication (Flament \& Rouquette, 2003; Rouquette, 1988; 1997; 1998a; 1998b; 1998c) des participants par rapport à l'initiative présentée (qui est ici l'objet par rapport auquel on mesure le niveau d'implication) devraient différer selon l'appartenance (nationale et religieuse) des participants et, de plus, nous pensons que les résultats obtenus devraient nous permettre de montrer l'indépendance respective de nos trois dimensions de l'implication.

\section{METHODES}

\section{Population}

Nous avons interrogé deux populations comparables: il s'agit d'étudiants de niveau universitaire, français $(n=81)$ et brésiliens $(n=73)$, tous fréquentant un cursus de Psychologie. L'âge moyen de ces étudiants (les deux nationalités confondues) est de 20,5 ans, avec un écart-type de 3,28 ans. De plus, chez les Français comme chez les Brésiliens, nous avons distingué deux sous-populations, sur la base de leur sentiment d'appartenance religieuse : un groupe d'étudiants se sentant catholiques et un groupe d'étudiants sans sentiment d'appartenance religieuse. Rappelons à ce propos que de ce point de vue, les deux pays sont comparables car ils sont, l'un et l'autre, de tradition catholique.

Cette étude interrogeait au total, dans un premier temps, deux cent neuf participants; par la suite, 55 participants ont été exclus de l'analyse car ils avaient un sentiment d'appartenance à des religions autres que la catholique. Les résultats ont donc été calculés, à la fin, sur un total de 154 participants (cf. tableau 1). Nous avons donc, en résumé, dans cette étude, deux variables indépendantes:

- L'appartenance nationale, à deux modalités : France versus Brésil.

- L'appartenance idéologique religieuse, à deux modalités: Appartenance religieuse catholique versus Sans appartenance religieuse.

En croisant les modalités de ces deux variables, on obtient le tableau d'effectifs suivant: 
Tableau 1. Répartition des Effectifs d'Etudiants par Groupe

\begin{tabular}{lccc}
\hline & \multicolumn{3}{c}{ Appartenance nationale } \\
\cline { 2 - 4 } Appartenance religieuse & Brésiliens & Français & Total \\
\hline Catholiques & 32 & 25 & 57 \\
Sans religion & 41 & 56 & 97 \\
\hline Total & 73 & 81 & 154 \\
\hline
\end{tabular}

La différence d'effectifs parmi les participants français, en ce qui concerne l'appartenance religieuse, s'explique par un plus vaste phénomène social, clairement mis en évidence dans les statistiques nationales (le nombre de personnes se déclarant catholiques a drastiquement baissé en France depuis 1990, en particulier chez les jeunes ${ }^{3}$ ). Au contraire, au Brésil, la religion catholique serait beaucoup plus suivie (chez les jeunes également) ${ }^{4}$ et ne connaîtrait pas de baisse remarquable.

Pour départager les participants du point de vue de l'appartenance religieuse, on s'est basés sur leurs réponses à la question: "Avez-vous le sentiment d'appartenir, ou de plutôt appartenir, à la religion...". Des choix multiples proposaient le choix : "Je n'ai pas le sentiment d'appartenir à une religion" et des choix correspondant à chacune des autres religions (seules les réponses des sujets déclarant un sentiment d'appartenance à la religion catholique ont été retenues). Pour départager les participants du point de vue de leur appartenance nationale, nous avons posé, dans le questionnaire, une question ouverte dans laquelle les participants devaient écrire leur nationalité. De plus, cela s'est fait en France et au Brésil et donc, le Pays de résidence des participants a, par ce biais, été apprécié (seuls les étudiants de nationalité française résidant en France et de nationalité brésilienne résidant au Brésil ont été retenus).

Le questionnaire a été présenté dans la langue locale. Enfin, pour tous les participants, les tests ont été collectifs et réalisés sur le terrain.

\section{L’inducteur: une "Dépêche" journalistique}

3 Cf. par ex. Lenoir (2007), aujourd'hui, les sondages indiquent que $51 \%$ des Français seulement se déclarent catholiques, quel que soit l'âge des participants.

4 Le dernier recensement général de la population, effectué en 2000 par l'IBGE (Institut Brésilien de Géographie et Statistiques), indiquait $74 \%$ de catholiques dans l'ensemble du Pays, quel que soit l'âge des participants. Ces chiffres restent, actuellement, à peu près stables.
Les participants devaient lire, sur une première page, un message qui leur était présenté comme une dépêche journalistique de cinq lignes. Ce message était présenté à l'identique à tous les participants.

Cette (fausse) dépêche est ambiguë par construction. Le message, présentant une initiative en faveur des sans-abris, lancée par l'Eglise, a, en effet, été construit en juxtaposant deux types d'information: premièrement, une distribution de repas gratuits aux sans-abris sur le parvis d'une église et deuxièmement, une invitation, toujours destinée aux mêmes sans-abris, à assister à un séminaire sur le thème "Être catholique aujourd'hui". Pour arriver à cette version définitive, 3 différents types de messages ont été testés sur 100 participants faisant partie de la population parente (toute appartenance religieuse confondue). Lors de ce pré-test, suite à la lecture du message, les participants devaient dire à quel point l'initiative présentée leur semblait "positive" ou "négative" (pour cela, deux échelles de Likert, allant de "Pas du tout positive" à "Tout à fait positive" et de "Pas du tout négative" à "Tout à fait négative" ont été présentées). Pour une seule des trois versions du message, la moyenne des scores pour ces deux échelles s'écartait d'un demipoint seulement du milieu de l'échelle: cela signifie que la moitié des participants a considéré le même texte comme étant positif et que l'autre moitié l'a considéré comme étant négatif. Ce texte peut donc être défini comme ambigu par construction. Cette version a donc été utilisée par la suite dans le questionnaire final et présentée sur la première page du questionnaire comme une dépêche "Reuters".

Après avoir lu la "dépêche", les participants devaient tourner la page et répondre aux questions traduisant les variables dépendantes (c'est-à-dire les trois échelles mesurant les dimensions indépendantes d'implication). A la fin du questionnaire, outre les questions signalétiques classiques (sexe, âge, etc.), des questions concernant les croyances et les pratiques religieuses étaient posées. Ces questions (et notamment celle relative au sentiment d'appartenance à une religion), ont permis de départager a posteriori les étudiants du point de vue de leurs appartenances religieuses.

\section{Mesures de l'implication.}

Pour les trois dimensions de l'implication (Flament \& Rouquette, 2003; Rouquette, 1988; 1997; 1998a; 1998b; 1998c) mesurées, à savoir la proximité, la mise en valeur de l'objet et la possibilité perçue d'action (PPA), les sujets répondaient à une question 
en se prenant position sur une échelle de Likert en 6 points.

La mesure de l'implication, étant donné les liens certains que cette dernière entretient avec les représentations sociales, devraient, vraisemblablement, nous renseigner également sur la nature et le niveau de structuration des représentations correspondantes, caractéristiques de nos groupes de participants. Néanmoins, l'analyse des liens intervenant entre représentations et implication ne constitue pas le but de cet article et, pour cela, nous renvoyons les lecteurs aux récents travaux sur ce thème (par ex. Baggio, 2006; Gruev-Vintila, 2005).

Il faut préciser, ici, que bien que nous fassions une référence à l'approche structurale des représentations sociales, notre objet (l'initiative présentée dans la "dépêche") ne peut être considéré comme un objet de représentation sociale à proprement parler. Mais, nous pensons que les résultats obtenus en mesurant l'implication sont en étroite liaison avec la nature et l'organisation des représentations rattachées au thème présenté par le message (l'aide fournie aux sans-abris par l'Eglise).

\section{Hypothèse}

Etant donné que les 3 dimensions sont indépendantes entre elles, nous pouvons émettre l'hypothèse qu'elles ne subiront pas le même effet que les variables indépendantes: chaque dimension sera influencée de façon différente par la nationalité, la religion et leur interaction.

\section{RESULTATS}

Pour la présentation des résultats, nous procéderons par dimension d'implication: d'abord, les résultats concernant la mise en valeur de l'objet, ensuite, les résultats concernant la possibilité perçue d'action et, enfin, les résultats concernant la proximité.

Pour chacune des dimensions seront mentionnés les effets simples de nos deux variables indépendantes, c'est-à-dire: (1) l'effet de l'appartenance religieuse (toutes nationalités confondues) et (2) l'effet de la nationalité (toutes appartenances religieuses confondues).

L'effet d'interaction entre l'appartenance religieuse et la nationalité sera également mentionné. Plus précisément, nous serons en présence d'un effet d'interaction lorsqu' une des deux variables n'agira pas de la même manière selon les modalités de l'autre variable.

\section{La mise en valeur de l'objet}

Les résultats obtenus sur la dimension "mise en valeur de l'objet" montrent que tous les groupes mettent en valeur l'événement d'une façon équivalente.

Tableau 2. Scores de Mise en valeur Selon la Nationalité et l'Appartenance Religieuse.

\begin{tabular}{lccc}
\hline Mise en Valeur & Brésil & France & Total \\
\hline Se sentant catholiques & 4.34 & 4.46 & 4.39 \\
Sans appartenance religieuse & 3.87 & 4.19 & 4.06 \\
\hline Total & 4.08 & 4.27 & \\
\hline
\end{tabular}

Cela signifie qu'indépendamment de l'appartenance religieuse et de la nationalité, tous les participants sont plutôt favorables à l'initiative. Ces résultats s'expliqueraient par une norme sociale: en effet, dans ces deux pays et, indépendamment de la religion, il serait socialement souhaitable "d'aider les gens", d'où le fait que tous les groupes valorisent l'initiative. Dans ce cas, nous sommes, en effet, en présence d'une manifestation de norme sociale supraordonnée par rapport aux différentes visions de la réalité dues aux appartenances groupales des individus. En d'autres termes, c'est bien d'aider autrui, que l'on soit brésilien ou français, que l'on soit catholique ou athé. Le fait qu'une telle norme soit aussi largement (et surtout socialement) souhaitable et partagée expliquerait, en somme, l'annulation des différences intergroupales observées.

\section{La possibilité perçue d'action (PPA).}

Les résultats obtenus sur la dimension "possibilité perçue d'action" illustrent le fait que catholiques et sans appartenance religieuse ont la même possibilité perçue d'action (test $t$ non significatif).

Tableau 3. Scores de Possibilité Perçue d'Action Selon la Nationalité et l'Appartenance Religieuse.

\begin{tabular}{lccc}
\hline PPA & Brésil & France & Total \\
\hline Se sentant catholiques & 1.91 & 3.20 & 2.46 \\
Sans appart.religieuse & 1.8 & 2.42 & 2.16 \\
\hline Total & 1.85 & 2.65 & \\
\hline
\end{tabular}

Contrairement à la variable indépendante "appartenance religieuse", la nationalité a un effet sur le sentiment de contrôle de la situation de la part des participants, les Français croyant pouvoir agir sur l'objet davantage que les Brésiliens; cette différence est significative (test $t(150)=4,20$ à $p .=.000045$. 
D'ailleurs, on remarque que l'appartenance religieuse ne joue un rôle sur la possibilité perçue d'action que chez les participants français; les Français catholiques ont en effet une PPA plus forte que les Français sans appartenance religieuse. Chez les Brésiliens, la religion ne joue, en revanche, aucun rôle sur cette dimension, catholiques et sans religion ayant la même PPA. Cela montre que nous sommes face à une interaction significative puisque la religion ne joue pas le même rôle selon les modalités de la variable nationalité (test $F(3 ; 148)=8,70 \quad p=.000024)$. Ce résultat semble indiquer que chez les participants Brésiliens, les différences en termes de possibilité perçue d'action ne dépendent pas de l'appartenance religieuse affirmée. Ils partageraient en particulier, plus que les Français, une idéologie de type catholique, prégnante même chez les non-croyants; cela constituerait, en particulier, une différence en termes d'effet de l'appartenance nationale entre nos deux populations.

\section{La proximité}

Les résultats concernant la proximité montrent que les participants se sentant catholiques se sentent plus proches de l'initiative présentée que ceux sans religion. Ce résultat est statistiquement significatif (test $t(151)=$ -2,88 à p.004).

Tableau 4. Scores de Proximité Selon la Nationalité et l'Appartenance Religieuse.

\begin{tabular}{lccc}
\hline Proximité & Brésil & France & Total \\
\hline Se sentant catholiques & 2.72 & 2.75 & 2.73 \\
Sans appart.religieuse & 2.04 & 2.07 & 2.06 \\
\hline Total & 2.24 & 2.25 & \\
\hline
\end{tabular}

Les participants se considérant catholiques se sentiraient plus proches de l'initiative car cette dernière émane de l'Eglise, ce résultat s'expliquerait, donc, par un sentiment d'identité groupale et de prises de positions particulières (cf. par ex. Poeschl, Campos et Ben Alaya, 2007), que n'auraient pas les participants non catholiques. La variable nationalité ne distingue en effet pas significativement (au test t) les participants sur la proximité à l'objet, Brésiliens et Français se sentant également proches de l'initiative. Ce cas est donc contraire par rapport au précédent: cette fois, lorsque la proximité à l'objet est mesurée (et non plus la possibilité perçue d'action), ce sont les différences d'appartenance nationale qui se trouvent neutralisées, et ce sont au contraire les différences en termes d'appartenance religieuse qui expliquent les différences trouvées. Ces résultats contribuent, une fois de plus, à montrer l'intérêt d'une utilisation conjointe des trois mesures d'implication qui permettent de nuancer les résultats.

\section{Synthèse des résultats sur les trois dimensions de l'implication personnelle}

Tableau 5. Tableau Récapitulatif des Effets des Variables Indépendantes sur les Trois Dimensions de l'Implication

\begin{tabular}{lccc}
\hline & $\begin{array}{c}\text { Effet simple } \\
\text { de } \\
\text { l'appartenanc } \\
\text { e religieuse }\end{array}$ & $\begin{array}{c}\text { Effet simple } \\
\text { de la } \\
\text { nationalité }\end{array}$ & $\begin{array}{c}\text { Interaction } \\
\text { nationalité } \mathbf{x} \\
\text { appartenance } \\
\text { religieuse }\end{array}$ \\
\hline Mise en Valeur & non & non & non \\
PPA & non & oui & oui \\
Proximité & oui & non & Non* \\
\hline
\end{tabular}

* L'interaction sur cette variable était légèrement significative, mais les moyennes sont proches, à un tel point qu'il n'a pas semblé pertinent de considérer que cette interaction avait lieu.

Les résultats précédents montrent que l'appartenance religieuse implique les participants sur une seule dimension de l'implication personnelle: la proximité par rapport à l'objet. Cette variable indépendante n'a pas d'influences significatives sur les deux autres dimensions; les participants sans religion et les participants se sentant catholiques ne se distinguent, ni sur la mise en valeur de l'objet, ni sur la possibilité perçue d'action.

L'autre variable indépendante (la nationalité) joue un rôle direct sur la possibilité perçue d'action: les Français se voient d'avantage comme des acteurs pouvant faire quelque chose que les Brésiliens. Par contre, la nationalité n'a pas d'effet significatif sur la mise en valeur de l'objet et la proximité, Français et Brésiliens donnent, en effet, la même importance à l'objet et se sentent aussi proches de l'initiative.

Enfin, les deux variables indépendantes interagissent sur la possibilité perçue d'action, puisque l'appartenance religieuse ne renforce la possibilité perçue d'action que chez les Français, les participants brésiliens ayant la même possibilité perçue d'action indépendamment de la religion.

Ces résultats montrent donc que (1) la mise en valeur de l'objet ne subit aucun effet de nos deux variables indépendantes, que (2) la proximité n'est influencée que par l'appartenance religieuse et que (3) la PPA est influencée par la nationalité et l'interaction entre la nationalité et l'appartenance religieuse.

\section{DISCUSSION}

Les résultats précédents illustrent bien le fait que l'implication personnelle distingue aussi bien les Brésiliens des Français ( $1^{\text {ère }}$ variable indépendante) que les participants sans appartenance religieuse des 
participants se sentant catholiques $\left(2^{\text {ème }}\right.$ variable indépendante). Cela souligne l'un des rôles majeurs de l'implication en tant qu'outil pour distinguer quantitativement les relations que différents groupes peuvent avoir avec un même objet. En d'autres termes, les quatre groupes expérimentaux se distinguent lorsqu' on mesure leur implication personnelle (et plus spécifiquement ses trois dimensions).

Il convient, aussi, de souligner que chaque dimension de l'implication subit un effet différent des variables indépendantes. Ainsi, la proximité subit l'effet de l'appartenance religieuse, alors que la PPA subit l'influence de la nationalité et de l'interaction entre les deux variables indépendantes. La mise en valeur de l'objet, quant à elle, se différencie des deux autres dimensions puisqu'elle ne subit aucune influence de la part des variables indépendantes. Ces résultats montrent clairement que les trois dimensions de l'implication sont indépendantes: si une dimension subit un effet, en aucun cas nous sommes en mesure d'affirmer qu'une des deux autres dimensions subira ce même effet. Par exemple, si les participants trouvent qu'un objet est important, cela ne traduit pas forcément une proximité forte. Une recherche empirique récente (Pecly Wolter \& Rouquette, 2006) illustre bien cela: des étudiants français trouvent que le tsunami a été un évènement très important (mise en valeur de l'objet forte), mais ne se sentent pas très concernés (proximité faible), le tsunami ayant eu lieu à des milliers de kilomètres de la France.

Comme nous espérons l'avoir montré au travers de cette étude, la mesure de l'implication personnelle, telle qu'elle a été opérationnalisée par Rouquette (1997), présente l'avantage de fournir des données particulièrement simples à traiter et "parlantes" (ces mesures sont présentées couramment sous forme d'échelles de Likert). Dans ce cadre, on décompose, en effet, le facteur d'implication personnelle des individus par rapport à l'objet en trois dimensions bien distinctes, ce qui permet d'apporter des nuances susceptibles d'être utiles à l'interprétation et, donc, d'introduire une certaine finesse dans les conclusions qui peuvent en être tirées.

Un autre avantage de ces mesures de l'implication, qui nous semble ressortir assez bien de cette étude, concerne le fait qu'elles peuvent être, en général, facilement comprises par les participants, ce qui les rend facilement applicables à des populations différentes : cela l'a déjà été démontré par des recherches récentes, dans lesquelles ces trois échelles, mesurant les différentes dimensions de l'implication, ont été utilisées sur une population française (Baggio, 2006; Gruev-Vintila, 2005), brésilienne (dans le cadre de cet article), italienne (à paraître) ou encore roumaine (Gruev-Vintila, 2005).
En dehors de ces qualités empiriques, certes indiscutables, mais pas suffisantes pour donner une réelle portée heuristique à ce facteur, nous pouvons en mettre en évidence d'autres, de plus grande portée théorique.

La psychologie sociale est une discipline plutôt bien armée pour étudier les relations entre une population et un objet. De la perception sociale (auteur, date) aux attitudes (auteur-date), plusieurs théories se penchent sur les liens entre un groupe et son environnement physique et social. Ce domaine de la psychologie est, d'après Moscovici, (1984) marqué par le regard ternaire et l'implication personnelle s'insère parfaitement dans cette triangulation, puisque la distance sujet-objet est forcément liée à la distance entre le groupe et l'objet. Sur cette distance entre le groupe et l'objet Flament et Rouquette (2003, p. 123) affirment que:

à l'intérieur de certaines limites, cette distance sera modulée par les événements qui se produisent, événements que l'on peut assimiler à des facteurs contextuels. Par exemple, la pollution atmosphérique m'affecte davantage en tant que thème et me concerne plus si un membre de ma famille proche est frappé de crises d'asthme imputables à une mauvaise qualité de l'air.

L'implication est donc davantage liée à des facteurs circonstanciels que les représentations sociales, qui varient très lentement et sont peu influencées par les contextes ponctuels. Un de ses atouts est qu'elle permet de saisir et prendre en compte, de manière opérationnelle, "les variations non structurales" (d'où sa complémentarité avec la théorie des représentations sociales).

L'implication, en tant que médiateur fondamental de la relation entre individu et objet, semble fournir, depuis déjà un certain temps en France, un outil précieux permettant la prise en compte de ce type de relation, et se constitue en même temps comme une variable explicative des modalités des liens individu-objet. L'implication personnelle se rattache, en effet, sur le plan théorique, au champ d'études des représentations sociales, qui, depuis longtemps déjà, ont connu un certain succès au Brésil ( par ex. Abric, 1998; 2003; Campos, 2003; Flament, 2001; Jodelet, 2001; Moscovici, 2003; Rateau, 2006; Sá, 1996; Vala, 1996).

Le facteur d'implication, qui agirait comme un médiateur des relations intervenant entre population et objet, permettrait notamment d'expliquer, du moins en bonne partie, les différences trouvées entre les représentations propres à des groupes sociaux distincts : ces différences peuvent, en effet, très souvent, être illustrées et expliquées par des différences de niveaux d'implication des participants constituant les groupes, que ces différences apparaissent sur la proximité à l'objet, sur la valorisation de 
l'objet ou encore sur la possibilité perçue d'action par rapport à l'objet.

Nous espérons, par conséquent, que cet article contribuera, d'une manière ou d'une autre, à la prise en compte de l'implication personnelle dans l'étude des représentations (Flament \& Rouquette, 2003), et à susciter à l'égard de ce facteur tout l'intérêt qu'il mérite.

\section{REFERÊNCIAS}

Abric, J.-C. (1998). A abordagem estrutural das representações sociais. Em A. S. P. Moreira \& D. C. de Oliveira (Eds.), Estudos interdisciplinares de representação social. (pp. 2738). Goiânia: AB.

Abric, J.-C. (2003). Abordagem estrutural das representações sociais: desenvolvimentos recentes. Em P. H. F. Campos \& M. C. da S. Loureiro (Orgs.), Representações sociais e práticas educativas (pp. 37-57). Goiânia: UCG.

Baggio, S. (2006). Pensée sociale et risques collectifs: Effets de l'implication personnelle sur la construction sociale des catastrophes naturelles. Unpublished thesis, Ecole doctorale 261, Cognitions, Comportements, Conduits Humaines, Université Paris Descartes, Paris.

Campos, P. H. F. (2003). A abordagem estrutural e o estudo das relações entre práticas e representações sociais. Em P. H. F. Campos \& M. C. da S. Loureiro (Orgs.), Representações sociais e práticas educativas (pp. 22-36). Goiânia: UCG.

Deconchy, J.-P. (1989). Psychologie Sociale, croyances et idéologies. Paris: Méridiens-Klinchsieck.

Flament, C. \& Rouquette, M.-L. (2003). Anatomie des idées ordinaire: Comment étudier les représentations sociales. Paris: Armand Colin.

Flament, C. (2001). Estrutura e dinâmica das representações sociais. Em D. Jodelet (Org.), As representações sociais (pp. 173-186). Rio de Janeiro: UERJ.

Gruev-Vintila, A. (2005). Dynamique de la représentation sociale d'un risque collectif et engagement dans les conduites de réduction du risque: Le rôle des pratiques, de l'implication et de la sociabilité. Unpublished thesis, Ecole doctorale 261, Cognitions, Université Paris Descartes, Paris.

Guimelli, C. (1998, Sep.). Implication, perception de la situation et représentations sociales: Étude expérimentale [Abstract]. En Actes du deuxième Colloque International de Psychologie Sociale en Langue Française (pp. 84-85), Turin.

Guimelli, C. (1999). La pensée sociale. Paris: Presses Universitaires de France.

Guimelli, C. (2002). Étude expérimentale du rôle de l'implication de soi dans les modalités de raisonnement intervenant dans le cadre des représentations sociales. Revue Internationale de Psychologie sociale, 15(1), 129-161.

Ibanez, T. (1999). Idéologie et relations inter groupes. En R.-Y. Bourhis \& J.-P. Leyens (Eds), Stéréotypes, discrimination et relations intergroupes (pp. 321-345). Sprimont, Belgique: Mardaga.

IBGE - Instituto Brasileiro de Geografia e Estatística (2000). Censo demográfico. Rio de Janeiro: IBGE.

Jodelet, D. (2001). Representações sociais: um domínio em expansão. In D. Jodelet (Ed.). As representações sociais (pp. 17-44). Rio de Janeiro: UERJ.

Lenoir, F. (2007, jan./fev.). Vers un catholicisme minoritaire? (Editorial) Le Monde des Religions, 21, 5.

Moscovici, S. (1984). Psychologie sociale. Paris: Presses Universitaires de France.

Moscovici, S. (2003). O fenômeno das representações sociais. In S. Moscovici (Org.). Representações sociais: investigações em psicologia social (pp. 29-109). Petrópolis: Vozes.

Pecly Wolter, R. \& Rouquette, M.-L. (2005). A influência de certos termos socialmente salientes (nexus) e da imagem sobre a percepção de um objeto social. Revista Educação Pública, 15(29), 79-89.

Poeschl, G., Campos, P. H. F.\& Ben Alaya, D. (2007). Appartenances nationales et prises de position sur la mondialisation, Bulletin de Psychologie, 60(1), 487, 11-19.

Rateau, P. (2006). Princípios organizadores e núcleo central das representações sociais. Hipóteses empíricas. Arquivos Brasileiros de Psicologia, 56(1-2), 93-104.

Rouquette, M.-L. (1988). La psychologie politique. Paris: Presses Universitaires de France.

Rouquette, M.-L. (1996). Représentations et idéologie. En J.C. Deschamps \& J.L. Beauvois (Eds), Des attitudes aux attributions (pp. 163-173). Grenoble: Presses Universitaires de Grenoble.

Rouquette, M.-L. (1997). La chasse à l'immigr: Violence, mémoire et représentations. Liège: Mardaga.

Rouquette, M.-L. (1998a). Les communications de masse. En S. Moscovici. La psychologie sociale. Paris: Presses Universitaires de France (First published in 1984).

Rouquette, M.-L. (1998b). La communication sociale. Paris: Dunod.

Rouquette, M.-L. (1998c). Sur la construction des mondes politiques. Bulletin de psychologie, 51(1), 433, 41-43.

Sá, C. P. de. (1996). Núcleo central das representações sociais. Petrópolis: Vozes.

Vala, J. (1996, $2^{\text {a }}$ Ed.). Representações sociais: para uma psicologia social do pensamento social. Em J. Vala \& M. B. Monteiro (Orgs.), Psicologia social (pp. 353-384). Lisboa: Calouste Gulbenkian.

Recebido em 31/05/2007

Aceito em 11/07/2007 


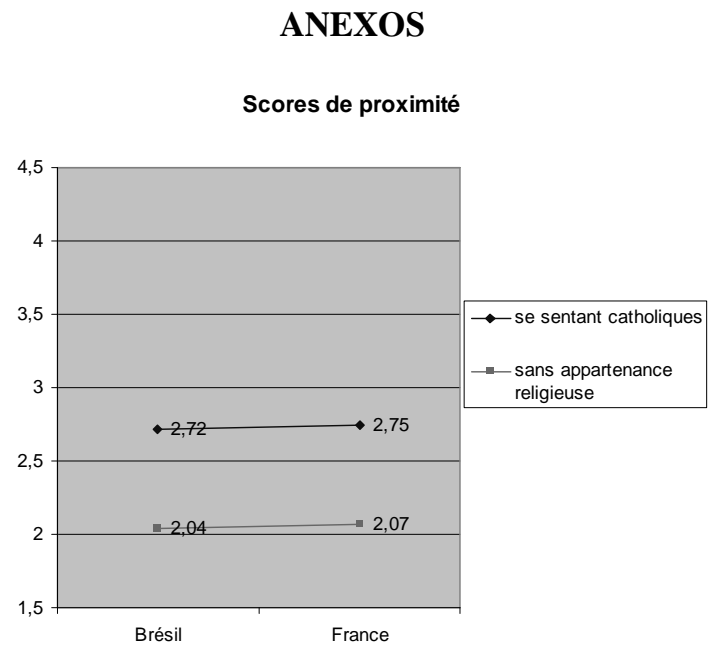

Graphique1. Scores Obtenus sur la Dimension de 1'Implication "Proximité"

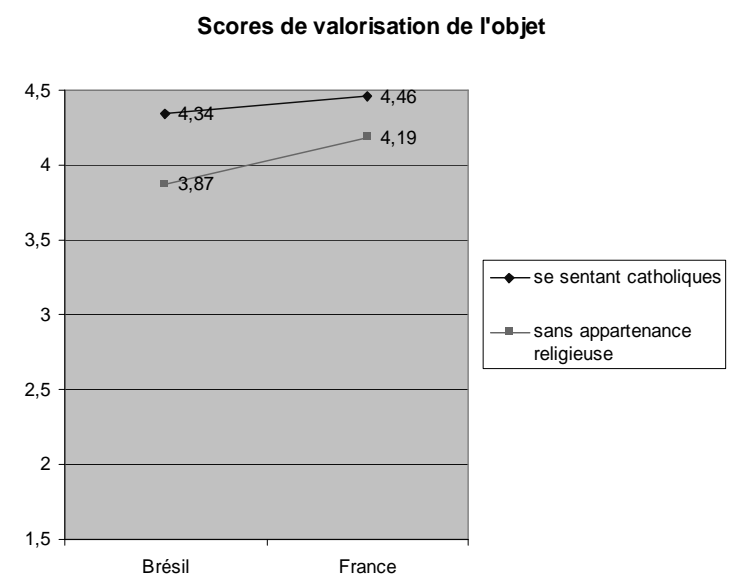

Graphique 2. Scores Obtenus sur la Dimension de l'Implication "Mise en Valeur de l'objet"

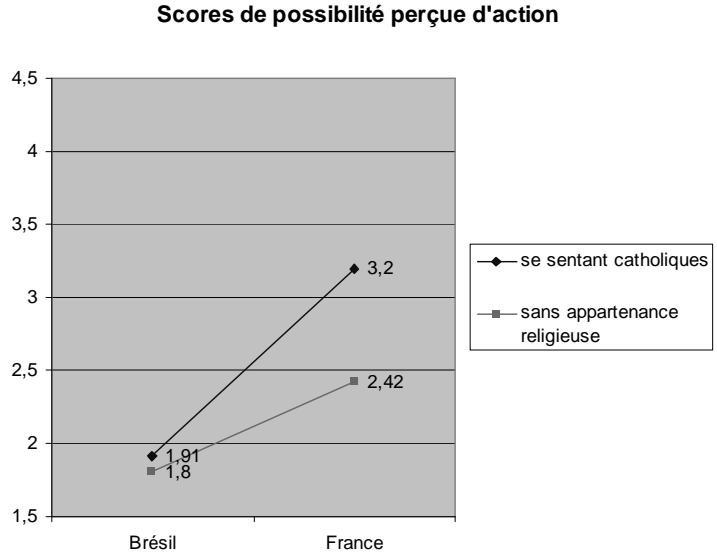

Graphique 3. Scores Obtenus sur la Dimension de l'Implication "possibilité perçue d'action". 